\title{
The Other Compartment: Challenges and Progress in Rectal Microbicide Development
}

\section{Peter A Anton* $*$}

\author{
Address: UCLA \\ Email: Peter A Anton* - panton@mednet.ucla.edu \\ * Corresponding author ‡Presenting author
}

\author{
from 2005 International Meeting of The Institute of Human Virology \\ Baltimore, USA, 29 August - 2 September 2005 \\ Published: 8 December 2005 \\ Retrovirology 2005, 2(Suppl I):S89 doi:I0.II86/I742-4690-2-SI-S89
}

Heterosexual RAI is common, with rates from $5-10 \%$ in the general population of women with up to $30-50 \%$ among women with other risk for HIV infection. RAI is considered to be the most common route of HIV transmission in men who have sex with men (MSM). Because of this broad and continuing HIV risk, topical HIV rectal microbicides are being developed to prevent rectal sexual transmission of HIV.

The environment of the rectosigmoid region of the colon is very different to the vagina, serving different physiological roles and, as a consequence, have unique and distinct features that complicate microbicide development. In contrast to the robust, stratified squamous, epithelium lining the vagina, the intestinal mucosa is lined by a single layer of columnar epithelium that is very vulnerable to trauma or potential microbicide toxicity. Moreover, the gut associated lymphoid tissue (GALT), underlying the mucosal surface, has a physiological level of inflammation perfectly suited to HIV replication. The limited anatomical space of the vaginal cavity contrasts with the relative enormity of the intestinal colon and raises profound questions about drug delivery and distribution. Despite these significant challenges, recent animal studies have shown the feasibility of preventing rectal SIV transmission using a locally applied microbicide.

Collaborative efforts are underway to begin to address these divergent needs in developing a safe rectal microbicide (RM). These include a recently concluded trial defining safety parameters (HPTN 056) and a focused NIH U19, now ending Year 1, with a targeted 5-year goal of initiating Phase 1 RM study. Failure to acknowledge the role that RAI plays in the global AIDS pandemic is likely to limit the success of intervention strategies and, more specifically, compromise attempts to develop safe and effective vaginal microbicides. 